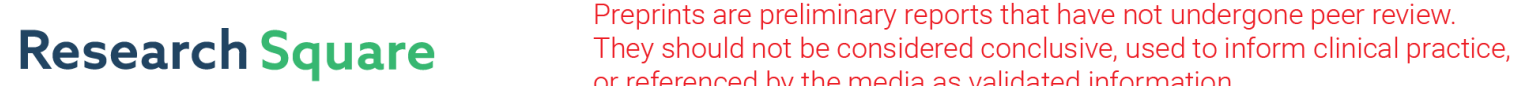 or referenced by the media as validated information. \\ CBD: A New Divergence Measure for Complex Mass Function and its Application in Pattern Recognition
}

\section{Lan Luo}

Southwest University

Fuyuan Xiao ( $\nabla$ doctorxiaofy@hotmail.com )

Southwest University https://orcid.org/0000-0002-4304-7189

\section{Research Article}

Keywords: Evidence theory, Complex mass function, Complex belief divergence, Uncertainty information, Pattern recognition

Posted Date: July 19th, 2021

DOl: https://doi.org/10.21203/rs.3.rs-579531/v1

License: (1) This work is licensed under a Creative Commons Attribution 4.0 International License. Read Full License 


\title{
CBD: A new divergence measure for complex mass function and its application in pattern recognition
}

\author{
Lan $\mathrm{Luo}^{\mathrm{a}}$, Fuyuan $\mathrm{Xiao}^{\mathrm{a}, *}$ \\ ${ }^{a}$ School of Computer and Information Science, Southwest University, Chongqing, 400715, China
}

\begin{abstract}
The theory of complex mass function is an effective method to deal with uncertainty information, and it is a generalized of Dempster-Shafer evidence theory. However, divergence measure is still an open issue in the realm of complex mass function theory. The main contribution of our paper is to propose a generalized divergence measure for complex mass function that is called complex belief divergence (CBD), which has the properties of symmetry, nonnegativity, nondegeneracy. When complex mass function degenerates into classical mass function, the CBD will degenerate into classical belief divergence, which has a better ability to measure uncertainty of information. Finally, a pattern recognition algorithm based on CBD is designed and applied to a medical diagnosis problem, which proves its practical prospect.
\end{abstract}

Keywords: Evidence theory, Complex mass function, Complex belief divergence, Uncertainty information, Pattern recognition

\footnotetext{
*Corresponding author: Fuyuan Xiao, School of Computer and Information Science, Southwest University, Chongqing, 400715, China.Email address: doctorxiaofy@hotmail.com.
} 


\section{Introduction}

Information is full of uncertainty in the real world [1-4], so it is important to develop the technology to deal with the uncertain information.Amounts of models and theories are proposed for uncertainty modeling, such as Dempster-Shafer evidence theory $[5,6], \mathrm{Z}$ numbers $[7,8]$, fuzzy sets theory [9-11], and other models [12-15]. Those models are widely used in real-world applications, including multi-criteria decision making [16-18], reliability analysis [19-22], classification [23, 24], complex network [25-27], medical diagnosis [28, 29], and risk analysis [30, 31].

Dempster-Shafer evidence theory is one of the most effective and efficient models to deal with uncertain information [32-34], and evidence theory is an extension to traditional probability theory as it assigns belief to multi-element subsets $[35,36]$. Hence, evidence theory has better ability to deal with uncertain information than traditional probability theory [37, 38].However, there are many open issues in Dempster-Shafer eviednce theory. For example, it can lead to counterintuitive results when the evidence is highly conflicting[39-41], how to determine the basic assignment[4244], how to measure the uncertainty of the evidence[45], and so on. Many applications are developed under the framework of Dempster-Shafer evidence theory [46-49]. Dempster-Shafer theory has also promoted the development of other theories, such as evidential reasoning[50-53], D numbers theory[54, 55], Pignistic belief transform[56, 57], and so on.

Therefore, a generalized Dempster-Shafer evidence theory is proposed[58, 59], which extends the classic Dempster-Shafer evidence theory to the complex form as a way of quantum processing. There are also many other models and methods for quantum information processing [60]. In this paper, a 
novel divergence measure called complex belief divergence (CBD) for complex mass function is proposed.

The following is the rest of paper organised. In Section 2, preliminaries include Dempster-Shafer evidence theory, complex Dempster-Shafer evidence theory and divergence measure in Dempster-Shafer theory will be briefly introduce. In Section 3, complex divergence measure and its properties will be discussed. Some numerical examples will be given in Section 4. And an application will be given in Section 5. In Section 6, conclusions will be made in the end.

\section{Preliminaries}

\subsection{Dempster-Shafer evidence theory}

Definition 2.1. The frame of discernment(FOD), a set of mutually collective and exclusive non-empty events $\Theta$, is defined below [61, 62]:

$$
\Theta=\left\{p_{1}, p_{2}, \ldots, p_{n}\right\}
$$

The power set of $\Theta$ is denoted as $2^{\Theta}$, and it is defined as:

$$
2^{\Theta}=\left\{\varnothing,\left\{p_{1}\right\},\left\{p_{1}, p_{2}\right\}, \ldots,\left\{p_{1}, p_{2}, \ldots, p_{n}\right\}\right\}
$$

where $\varnothing$ is an empty set.

Definition 2.2. The FOD $\Theta$ must satisfy the following properties [61, 62]:

$$
\begin{aligned}
& \text { (1) } m(\varnothing)=0 \\
& \text { (2) } \sum_{A \in 2^{\Theta}} m(A)=1,
\end{aligned}
$$

where $m: 2^{\Theta} \rightarrow[0,1]$ is called the basic probability assignment $(B P A)$, which is 
also known as the mass function. And if $m(A)>0, A \in 2^{\Theta}$, then $A$ is called the focal element.

The classic Dempster combination rule (DCR) is defined by Dempster to combine different evidences, and it is given as follows [61].

Definition 2.3. Given two independent BPAs, $m_{1}, m_{2}$, the obtained BPA of DCR can be denoted as $m$, and it can be obtained as follows:

$$
\left\{\begin{array}{l}
m(\varnothing)=0 \\
m(A)=\frac{\sum_{B \cap C=A} m_{1}(B) m_{2}(C)}{1-K}
\end{array}\right.
$$

where $K=\sum_{B \cap C=\varnothing} m_{1}(B) m_{2}(C)$ and $K<1$.

\subsection{Complex Dempster-Shafer evidence theory}

Complex Dempster-Shafer evidence theory is the generalization of DempsterShafer evidence theory as it is defined on complex numbers [58, 59].

Definition 2.4. A set of mutually exclusive and collective non-empty events $\Theta$ is called the frame of discernment (FOD). It is defined as follows:

$$
\Theta=\left\{p_{1}, p_{2}, \ldots, p_{n}\right\}
$$

The power set of $\Theta$ is denoted as $2^{\Theta}$, and it is defined as:

$$
2^{\Theta}=\left\{\varnothing,\left\{p_{1}\right\},\left\{p_{1}, p_{2}\right\}, \ldots,\left\{p_{1}, p_{2}, \ldots, p_{n}\right\}\right\}
$$

where $\varnothing$ is an empty set. 
Definition 2.5. A complex mass function $\mathbb{M}$ in $F O D \Theta$ is represented as a mapping from $2^{\Theta} \rightarrow \mathbb{C}$, defined by

$$
\mathbb{M}: 2^{\Theta} \rightarrow \mathbb{C}
$$

M satisfies following properties.

1. $\mathbb{M}(\varnothing)=0$

2. $\mathbb{M}(A)=m(A) e^{i \theta(A)}, A \in 2^{\Theta}$.

3. $\sum_{A \in 2^{\Theta}} \mathbb{M}(A)=1$

where $i=\sqrt{-1} . m(A)$ represents the magnitude of $\mathbb{M}(A)$ while $\theta(A)$ represents the phase of $\mathbb{M}(A) . m(A) \in[0,1], \theta(A) \in\left[-\frac{\pi}{2}, \frac{\pi}{2}\right][63]$.

The following is another expression of $\mathbb{M}$.

\section{Definition 2.6.}

$$
\mathbb{M}(A)=x+y i, A \in 2^{\Theta}
$$

which satisfies $\sqrt{x^{2}+y^{2}} \leq 1$. Also, $m(A)=\sqrt{x^{2}+y^{2}}$ and $\theta(A)=\arctan \left(\frac{y}{x}\right)$.

A is a focal element satisfying $|\mathbb{M}(A)|>0$ and $A \in 2^{\Theta}$. When all $\mathbb{M}(\mathrm{A})$ is real number, complex Dempster-Shafer evidence theory degenerates into classical Dempster-Shafer evidence theory.

Definition 2.7. Suppose there are two independent complex mass functions, $\mathbb{M}_{\mathbb{1}}$, $\mathbb{M}_{2}$, the obtained BPA of DCR can be denoted as $\mathbb{M}$, and it can be obtained as follows:

$$
\left\{\begin{array}{l}
\mathbb{M}_{1}(\varnothing)=0 \\
\mathbb{M}_{1}(A)=\frac{\sum_{\cap=A} \mathbb{M}_{1}(B) \mathbb{M}_{2}(C)}{1-K}
\end{array}\right.
$$

where $K=\sum_{B \cap C=\varnothing} \mathbb{M}_{1}(B) \mathbb{M}_{2}(C)$ and $K<1$. 


\subsection{Divergence measure in Dempster-Shafer evidence theory}

Divergence is useful for difference measure of information [64-66], which has been used in many fields [67-70]. For example, relative belief entropy [71] is proposed by Fei and Deng based on Kullback-Leibler divergence [72].

Definition 2.8. Given two mass functions $m_{1}$ and $m_{2}$, the definition of relative belief entropy is as follows.

$$
K L\left(m_{1} \| m_{2}\right)=\sum_{A \in 2^{\Theta}} m_{1}(A) \log \left(\frac{m_{1}(A)}{m_{2}(A)}\right)
$$

It should be noted that when $m_{1}(A)=0, m_{1}(A) \log \left(\frac{m_{1}(A)}{m_{2}(A)}\right)$ should be interpreted as 0 . Also, it can be proved that $K L\left(m_{1} \| m_{2}\right)>0$. In this paper, a natural number e is taken for all log functions.

Definition 2.9. Jensen-Shannon belief divergence is defined by Fei and Deng as a distance measure in belief theory [71], and it is presented as follows.

$$
\begin{aligned}
J S\left(m_{1} \| m_{2}\right) & =\frac{1}{2} K L\left(m_{1} \| \frac{m_{1}+m_{2}}{2}\right)+\frac{1}{2} K L\left(m_{2} \| \frac{m_{1}+m_{2}}{2}\right) \\
& =\frac{1}{2} \sum_{A \in 2^{\Theta}}\left(m_{1}(A) \log \frac{2 m_{1}(A)}{m_{1}(A)+m_{2}(A)}\right) \\
& +\frac{1}{2} \sum_{A \in 2^{\Theta}}\left(m_{2}(A) \log \frac{2 m_{2}(A)}{m_{1}(A)+m_{2}(A)}\right)
\end{aligned}
$$

Inspired by belief entropy [73, 74], Song and Deng improve the relative divergence by considering the uncertainty caused by subsets [75], and the improved relative divergence is given as follows. 
Definition 2.10.

$$
K L\left(m_{1} \| m_{2}\right)=\sum_{A \in 2^{\Theta}} \frac{m_{1}(A)}{2^{|A|}-1} \log \left(\frac{m_{1}(A)}{m_{2}(A)}\right)
$$

When all focal elements $A$ are single elements, Eq.(11) degenerates into Eq.(9).

Based on improved relative divergence, the definition of the improved Jensen-Shannon belief entropy is as follows.

\section{Definition 2.11.}

$$
\begin{aligned}
J S\left(m_{1} \| m_{2}\right) & =\frac{1}{2} K L\left(m_{1} \| \frac{m_{1}+m_{2}}{2}\right)+\frac{1}{2} K L\left(m_{2} \| \frac{m_{1}+m_{2}}{2}\right) \\
& =\frac{1}{2} \sum_{A \in 2^{\Theta}} \frac{1}{2^{|A|}-1}\left(m_{1}(A) \log \frac{2 m_{1}(A)}{m_{1}(A)+m_{2}(A)}\right) \\
& +\frac{1}{2} \sum_{A \in 2^{\Theta}} \frac{1}{2^{|A|}-1}\left(m_{2}(A) \log \frac{2 m_{2}(A)}{m_{1}(A)+m_{2}(A)}\right)
\end{aligned}
$$

\section{Complex belief divergence}

In this section, we propose a new divergence measure called CBD. The new measure extends from a real number system to a complex number system and also takes into account the cardinality of subsets.

\subsection{Definition of $C B D$}

Based on the relative belief entropy improved by Song and Deng [75], the proposed relative entropy is extended to the complex number system.

Definition 3.1. There are two complex mass functions $\mathbb{M}_{1}$ and $\mathbb{M}_{2}$, and the definition of complex relative belief entropy is as

$$
K L\left(\mathbb{M}_{1}|| \mathbb{M}_{2}\right)=\sum_{A \in 2^{\Theta}} \frac{1}{2^{|A|}-1}\left(\left|\mathbb{M}_{1}(A)\right| \log _{2} \frac{\left|\mathbb{M}_{1}(A)\right|}{\left|\mathbb{M}_{2}(A)\right|}\right)
$$


And improved Jensen-Shannon belief divergence is extended to the complex number system as complex belief divergence (CBD) as follows:

Definition 3.2. There are two complex mass functions $\mathbb{M}_{1}$ and $\mathbb{M}_{2}$. The definition of the complex belief divergence (CBD) JS $\left(\mathbb{M}_{1}|| \mathbb{M}_{2}\right)$ ) is follows.

$$
\begin{aligned}
J S\left(\mathbb{M}_{1}|| \mathbb{M}_{2}\right) & =\frac{1}{2} \sum_{A \in 2^{\Theta}} \frac{1}{2^{|A|}-1}\left(\left|\mathbb{M}_{1}(A)\right| \log _{2} \frac{2\left|\mathbb{M}_{1}(A)\right|}{\left|\mathbb{M}_{1}(A)+\mathbb{M}_{2}(A)\right|}\right) \\
& +\frac{1}{2} \sum_{A \in 2^{\Theta}} \frac{1}{2^{|A|}-1}\left(\left|\mathbb{M}_{2}(A)\right| \log _{2} \frac{2\left|\mathbb{M}_{2}(A)\right|}{\left|\mathbb{M}_{1}(A)+\mathbb{M}_{2}(A)\right|}\right)
\end{aligned}
$$

\subsection{Properties of $C B D$}

Theorem 3.1. JS $\left(\mathbb{M}_{1} \| \mathbb{M}_{2}\right)$ is symmetric. The $C B B A s, \mathbb{M}_{1}$ and $\mathbb{M}_{2}$, are two BPAs mapping to complex numbers, and JS is the divergence of the two CBBAs, then we get: $J S\left(\mathbb{M}_{1} \| \mathbb{M}_{2}\right)=J S\left(\mathbb{M}_{2}|| \mathbb{M}_{1}\right)$.

Proof. According to Eq.(10) we have:

$$
\begin{aligned}
& J S\left(\mathbb{M}_{1} \| \mathbb{M}_{2}\right)=\frac{1}{2} K L\left(\mathbb{M}_{1} \| \frac{\mathbb{M}_{1}+\mathbb{M}_{2}}{2}\right)+\frac{1}{2} K L\left(\mathbb{M}_{2} \| \frac{\mathbb{M}_{1}+\mathbb{M}_{2}}{2}\right) \\
& J S\left(\mathbb{M}_{2} \| \mathbb{M}_{1}\right)=\frac{1}{2} K L\left(\mathbb{M}_{2} \| \frac{\mathbb{M}_{1}+\mathbb{M}_{2}}{2}\right)+\frac{1}{2} K L\left(\mathbb{M}_{1} \| \frac{\mathbb{M}_{1}+\mathbb{M}_{2}}{2}\right)
\end{aligned}
$$

so,in any case $J S\left(\mathbb{M}_{1}|| \mathbb{M}_{2}\right)=J S\left(\mathbb{M}_{2}|| \mathbb{M}_{1}\right)$

Theorem 3.2. $J S\left(\mathbb{M}_{1}|| \mathbb{M}_{2}\right)$ is nonnegative. The $C B B A s, \mathbb{M}_{1}$ and $\mathbb{M}_{2}$, are two BPAs mapping to complex numbers and JS is the divergence of the two CBBAs. It comes to a conclusion that $J S\left(\mathbb{M}_{1}|| \mathbb{M}_{2}\right) \geq 0$.

Proof. According to the knowledge that $x-1 \geq \log _{2}(x)$ when $x \in(0,1]$, 
we have:

$$
\begin{aligned}
-K L & \left(\mathbb{M}_{1}(A)|| \frac{\mathbb{M}_{1}(A)+\mathbb{M}_{2}(A)}{2}\right) \\
& =-\sum_{A \in 2^{\Theta}} \frac{1}{2^{|A|}-1}\left(\left|\mathbb{M}_{1}(A)\right| \log _{2} \frac{2\left|\mathbb{M}_{1}(A)\right|}{\left|\mathbb{M}_{1}(A)+\mathbb{M}_{2}(A)\right|}\right) \\
& =\sum_{A \in 2^{\Theta}} \frac{1}{2^{|A|}-1}\left(\left|\mathbb{M}_{1}(A)\right| \log _{2} \frac{\left|\mathbb{M}_{1}(A)+\mathbb{M}_{2}(A)\right|}{2\left|\mathbb{M}_{1}(A)\right|}\right) \\
& \leq \sum_{A \in 2^{\Theta}} \frac{\left|\mathbb{M}_{1}(A)\right|}{2^{|A|}-1}\left(\frac{\left|\mathbb{M}_{1}(A)+\mathbb{M}_{2}(A)\right|}{2\left|\mathbb{M}_{1}(A)\right|}-1\right) \\
& =\sum_{A \in 2^{\Theta}} \frac{\left|\mathbb{M}_{1}(A)\right|}{2^{|A|}-1}\left(\frac{\left|\mathbb{M}_{1}(A)+\mathbb{M}_{2}(A)\right|-2\left|\mathbb{M}_{1}(A)\right|}{2\left|\mathbb{M}_{1}(A)\right|}\right) \\
& =\sum_{A \in 2^{\Theta}} \frac{1}{2^{|A|}-1}\left(\frac{\left|\mathbb{M}_{1}(A)+\mathbb{M}_{2}(A)\right|-2\left|\mathbb{M}_{1}(A)\right|}{2}\right)
\end{aligned}
$$

To prove in the same way, we can get:

$$
-K L\left(\mathbb{M}_{2}(A)|| \frac{\mathbb{M}_{1}(A)+\mathbb{M}_{2}(A)}{2}\right)=\sum_{A \in 2^{\Theta}} \frac{1}{2^{|A|}-1}\left(\frac{\left|\mathbb{M}_{1}(A)+\mathbb{M}_{2}(A)\right|-2\left|\mathbb{M}_{2}(A)\right|}{2}\right)
$$

Then, sum the Eq.(17) and Eq.(18).

$$
\begin{aligned}
& K L\left(\mathbb{M}_{1}(A)|| \frac{\mathbb{M}_{1}(A)+\mathbb{M}_{2}(A)}{2}\right)+K L\left(\mathbb{M}_{2}(A)|| \frac{\mathbb{M}_{1}(A)+\mathbb{M}_{2}(A)}{2}\right) \\
& \geq \sum_{A \in 2^{\Theta}} \frac{1}{2^{|A|}-1}\left(\left|\mathbb{M}_{1}(A)\right|+\left|\mathbb{M}_{2}(A)\right|-\left|\mathbb{M}_{1}(A)+\mathbb{M}_{2}(A)\right|\right)
\end{aligned}
$$

And we assume that there are two complex numbers $\mathbb{M}_{1}=a+b i$ and 


$$
\begin{aligned}
& \mathbb{M}_{2}=c+d i, \text { it is easily to prove that }\left|\mathbb{M}_{1}\right|+\left|\mathbb{M}_{2}\right| \geq\left|\mathbb{M}_{1}+\mathbb{M}_{2}\right| \\
& \left|\mathbf{M}_{1}\right|+\left|\mathbb{M}_{2}\right|-\left|\mathbb{M}_{1}+\mathbb{M}_{2}\right|=|a+b i|+|c+d i|-|(a+c)+(b+d) i| \\
& =\sqrt{a^{2}+b^{2}}+\sqrt{c^{2}+d^{2}}-\sqrt{(a+c)^{2}+(b+d)^{2}} \\
& =\frac{\left(\sqrt{a^{2}+b^{2}}+\sqrt{c^{2}+d^{2}}\right)^{2}-\left(\sqrt{(a+c)^{2}+(b+d)^{2}}\right)^{2}}{\sqrt{a^{2}+b^{2}}+\sqrt{c^{2}+d^{2}}+\sqrt{(a+c)^{2}+(b+d)^{2}}} \\
& =\frac{a^{2}+b^{2}+c^{2}+d^{2}+2 \sqrt{a^{2}+b^{2}} \sqrt{c^{2}+d^{2}}-\left((a+c)^{2}+(b+d)^{2}\right)}{\sqrt{a^{2}+b^{2}}+\sqrt{c^{2}+d^{2}}+\sqrt{(a+c)^{2}+(b+d)^{2}}} \\
& =\frac{2\left(\sqrt{a^{2}+b^{2}} \sqrt{c^{2}+d^{2}}-a c-b d\right)}{\sqrt{a^{2}+b^{2}}+\sqrt{c^{2}+d^{2}}+\sqrt{(a+c)^{2}+(b+d)^{2}}} \\
& =\frac{2\left(\sqrt{a^{2}+b^{2}} \sqrt{c^{2}}+d^{2}-a c-b d\right)\left(\sqrt{a^{2}+b^{2}} \sqrt{c^{2}+d^{2}}+a c+b d\right)}{\left(\sqrt{a^{2}+b^{2}}+\sqrt{c^{2}+d^{2}}+\sqrt{(a+c)^{2}+(b+d)^{2}}\right)\left(\sqrt{a^{2}+b^{2}} \sqrt{c^{2}+d^{2}}+a c+b d\right)} \\
& =\frac{2\left(a^{2} d^{2}+b^{2} c^{2}\right)-2 a b c d}{\left(\sqrt{a^{2}+b^{2}}+\sqrt{c^{2}+d^{2}}+\sqrt{(a+c)^{2}+(b+d)^{2}}\right)\left(\sqrt{a^{2}+b^{2}} \sqrt{c^{2}+d^{2}}+a c+b d\right)} \\
& =\frac{2(a d-b c)^{2}}{\left(\sqrt{a^{2}+b^{2}}+\sqrt{c^{2}+d^{2}}+\sqrt{(a+c)^{2}+(b+d)^{2}}\right)\left(\sqrt{a^{2}+b^{2}} \sqrt{c^{2}+d^{2}}+a c+b d\right)} \\
& \geq 0
\end{aligned}
$$

So,we have $\left|\mathbb{M}_{1}\right|+\left|\mathbb{M}_{2}\right|-\left|\mathbb{M}_{1}+\mathbb{M}_{2}\right| \geq 0$,and use this in Eq.(17).So, we have $J S\left(\mathbb{M}_{1}|| \mathbb{M}_{2}\right) \geq 0$.

Theorem 3.3. The CBBAs, $\mathbb{M}_{1}$ and $\mathbb{M}_{2}$, are two BPAs mapping to complex numbers and JS is the divergence of the two CBBAs. It comes to a conclusion that $J S\left(\mathbb{M}_{1}|| \mathbb{M}_{2}\right)=0$ only if $\mathbb{M}_{1}$ and $\mathbb{M}_{2}$ have same real part and imaginary part.

Proof.

$$
\begin{aligned}
-K L & \left(\mathbb{M}_{1}(A) \| \frac{\mathbb{M}_{1}(A)+\mathbb{M}_{2}(A)}{2}\right) \\
& =\sum_{A \in 2^{\Theta}} \frac{1}{2^{|A|}-1}\left(\frac{\left|\mathbb{M}_{1}(A)+\mathbb{M}_{2}(A)\right|-2\left|\mathbb{M}_{1}(A)\right|}{2}\right)
\end{aligned}
$$


Because $\mathbb{M}_{1}(A)$ and $\mathbb{M}_{2}(A)$ are equal, so $\left|\mathbb{M}_{1}(A)+\mathbb{M}_{2}(A)\right|=2\left|\mathbb{M}_{1}(A)\right|$ and $J S\left(\mathbb{M}_{1}|| \mathbb{M}_{2}\right)=0$.

\section{Numerical examples}

Example 4.1. Suppose that $X=\{A, B, C\}$ is a frame of discernment. There are two mass functions as follows:

1. $\mathbb{M}_{1}(A)=0.4+0.2 i, \mathbb{M}_{1}(B)=0.3+0.2 i, \mathbb{M}_{1}(C)=0.3-0.4 i$;

$$
\mathbb{M}_{2}(A)=0.4+0.2 i, \mathbb{M}_{2}(B)=0.3+0.2 i, \mathbb{M}_{2}(C)=0.3-0.4 i ;
$$

2. $\mathbb{M}_{1}(A, B)=0.4+0.2 i, \mathbb{M}_{1}(A, C)=0.6-0.2 i$;

$$
\mathbb{M}_{2}(A, B)=0.4+0.2 i, \mathbb{M}_{2}(A, C)=0.6-0.2 i ;
$$

The calculation process of divergence are as follows:

1. $J S\left(\mathbb{M}_{1}|| \mathbb{M}_{2}\right)=\frac{1}{2} \sum_{A \in 2^{\Theta}} \frac{1}{2^{|A|}-1}\left(\left|\mathbb{M}_{1}(A)\right| \log _{2} \frac{2\left|\mathbb{M}_{1}(A)\right|}{\left|\mathbb{M}_{1}(A)+\mathbb{M}_{2}(A)\right|}\right)$

$+\frac{1}{2} \sum_{A \in 2^{\Theta}} \frac{1}{2^{|A|}-1}\left(\left|\mathbb{M}_{2}(A)\right| \log _{2} \frac{2\left|\mathbb{M}_{2}(A)\right|}{\left|\mathbb{M}_{1}(A)+\mathbb{M}_{2}(A)\right|}\right)$

$=\frac{1}{2} \times \frac{1}{2^{1}-1} \times \sqrt{0.4^{2}+0.2^{2}} \times \log _{2} \frac{2 \times \sqrt{0.4^{2}+0.2^{2}}}{\sqrt{(0.4+0.4)^{2}+(0.2+0.2)^{2}}}$

$+\frac{1}{2} \times \frac{1}{2^{1}-1} \times \sqrt{0.3^{2}+0.2^{2}} \times \log _{2} \frac{2 \times \sqrt{0.3^{2}+0.2^{2}}}{\sqrt{(0.3+0.3)^{2}+(0.2+0.2)^{2}}}$

$+\frac{1}{2} \times \frac{1}{2^{1}-1} \times \sqrt{0.3^{2}+0.4^{2}} \times \log _{2} \frac{2 \times \sqrt{0.3^{2}+0.4^{2}}}{\sqrt{(0.3+0.3)^{2}+(0.4+0.4)^{2}}}$

$=0$

2. $J S\left(\mathbb{M}_{1}|| \mathbb{M}_{2}\right)=\frac{1}{2} \sum_{A \in 2^{\Theta}} \frac{1}{2^{|A|}-1}\left(\left|\mathbb{M}_{1}(A)\right| \log _{2} \frac{2\left|\mathbb{M}_{1}(A)\right|}{\left|\mathbb{M}_{1}(A)+\mathbb{M}_{2}(A)\right|}\right)$

$+\frac{1}{2} \sum_{A \in 2^{\Theta}} \frac{1}{2^{|A|}-1}\left(\left|\mathbb{M}_{2}(A)\right| \log _{2} \frac{2\left|\mathrm{M}_{2}(A)\right|}{\left|\mathbb{M}_{1}(A)+\mathbb{M}_{2}(A)\right|}\right)$

$=\frac{1}{2} \times \frac{1}{2^{2}-1} \times \sqrt{0.4^{2}+0.2^{2}} \times \log _{2} \frac{2 \times \sqrt{0.4^{2}+0.2^{2}}}{\sqrt{(0.4+0.4)^{2}+(0.2+0.2)^{2}}}$

$+\frac{1}{2} \times \frac{1}{2^{2}-1} \times \sqrt{0.6^{2}+0.2^{2}} \times \log _{2} \frac{2 \times \sqrt{0.6^{2}+0.2^{2}}}{\sqrt{(0.6+0.6)^{2}+(0.2+0.2)^{2}}}$

$=0$

From the cases of Example 4.1, it gains a conclusion that the divergence is zero when two CBBAs have the same real part and imaginary part.And also it proves 
that the relative divergence improved by Song and Deng considering the uncertainty caused by subsets [75]can degenerate into the relative divergence without considering the number of elements in the subsets.

Example 4.2. Set that there are two $C B B A$ s below:

$\mathbb{M}_{1}(A): \mathbb{M}_{1}(A)=x+y i, \mathbb{M}_{1}\left(X_{\Theta}\right)=1-x-y i$,

$\mathbb{M}_{2}(A): \mathbb{M}_{2}(A)=1-x+y i, \mathbb{M}_{2}\left(X_{\Theta}\right)=x-y i$

where $\Theta \in\{1,2\} \cdot X_{1}=\{B\}$ and $X_{2}=\{A, B\}$ respectively when $\Theta=1$ and $\Theta=2$. The values of $\mathbb{M}_{1}(A)$ and $\mathbb{M}_{2}(A)$ vary from the variables $x$ and $y$.

Suppose $y=0$, so the $C B B A$ s $\mathbb{M}_{1}(A)$ and $\mathbb{M}_{2}(A)$ degenerate into classical $B$ $B A s$. The JS divergence change from the variable $x$ within the range $[0,1]$ depicted in Fig.1(a). The solid line represents $\{B\}$ and the dashed line represents $\{A, B\}$. This finding verifies that when the complex number degenerate into real number, the divergence measure works.

Suppose $x$ within the range of $[0,1]$ and $y=0.1$, the two $C B B A s \mathbb{M}_{1}(A)$ and $\mathrm{M}_{2}(A)$ are complex numbers. The value of JS divergences with the variation of $x$ in the range of $[0,1]$, in the case of the singleton and multiple subsets, is depicted in Fig.1(b). For another case, the divergence is 0 no matter what kind of sets the $C B B A$ s are when $x=0.5$ and $y=0.1$. And for the case of $x$ in the range of $[0,0.5) \cup(0.5,1]$, even if $\mathbb{M}_{1}(A)$ and $\mathbb{M}_{2}(A)$ have the same value, the divergence between $\mathbb{M}_{1}(A)$ and $\mathbb{M}_{2}(A)$ in the case of $X_{1}=\{B\}$ is bigger than the case of $X_{2}=\{A, B\}$.The reason for intuitive and reasonable result is that $X_{2}=\{A, B\}$ has intersection with $\{A\}$.

In addition, from the two results showed in Fig.1, it can gain a conclusion that the divergence is symmetrical and nonnegative.

Example 4.3. Set that there are two $C B B A$ s in $\omega=\{A, B\}$ below:

$\mathbb{M}_{1}(A): \mathbb{M}_{1}(A)=x+y i, \mathbb{M}_{1}(\{B\})=1-x-y i$, 


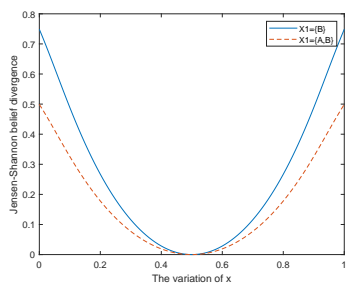

(a) JS between real sets

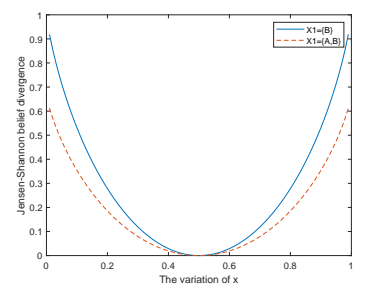

(b) JS between complex sets

Figure 1: JS divergence in Example 4.2

$\mathbb{M}_{2}(A): \mathbb{M}_{2}(A)=1-x+(y+a) i, \mathbb{M}_{2}(\{B\})=x+(a-y) i$.

The values of $\mathbb{M}_{1}(A)$ and $\mathbb{M}_{2}(A)$ vary from the parameters $x$ and $y$.

To study the value of $C B D$ how to change with the CBBAs in the real and imaginary part, there are all four kinds of different situations to discuss. Suppose two variables $x y$ respectively in the range of $[0,1]$ and $[-1,1]$, meeting the condition that $x^{2}+y^{2} \leq 1,(1-x)^{2}+y^{2} \leq 1,(1-x)^{2}+(y-a)^{2} \leq 1$ and $(1-x)^{2}+(a-y)^{2} \leq 1$.

When we set $a=0$,two $C B B A s \mathbb{M}_{1}(A)$ and $\mathbb{M}_{2}(A)$ have the same imaginary part in the Example 4.2. With $x$, the real part, in the range of $[0,1]$ in the Fig.2(a), the CBD has the minimum value of zero when $x=0.5$, exactly two $C B$ $B$ As have the same real part, such that $\mathbb{M}_{1}(A)$ is equal to $\mathbb{M}_{2}(A)$. In Fig.2(b), the JS divergence is greater than zero in other cases of $x$ and $y$, because of the different imaginary parts between $\mathbb{M}_{1}(A)$ and $\mathbb{M}_{2}(A)$. Moreover, Fig.2(c) shows that as $x$ change from 0 to 0.5 , the JS divergence decreases, because two CBBAs gradually become similar. On the contrast, when $x$ change 0.5 to 1, the JS divergence increase.

When we set $y=0.1$ and $a$ in the range of $[-1,1]$,two $C B B A s \mathbb{M}_{1}(A)$ and $\mathbb{M}_{2}(A)$ have different imaginary part, we have $\mathbb{M}_{1}(A)=x+0.1 i, \mathbb{M}_{2}(B)=$ $1-x-0.1 i$ and $\mathbb{M}_{1}(A)=x+(0.1-a) i, \mathbb{M}_{2}(B)=1-x-(0.1-a)$ i. The 


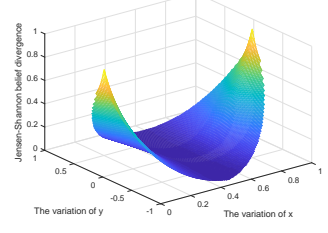

(a) The value of JS

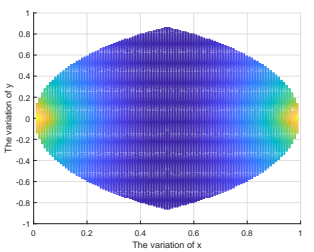

(b) Variations in $\mathrm{x}$ and $\mathrm{y}$

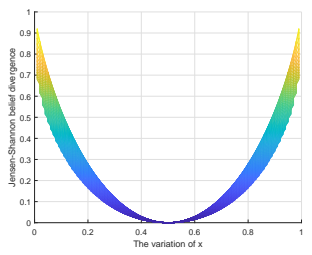

(c) JS as x varies

Figure 2: JS as CBBAs vary in the real part

variation of JS divergence, as the variable a within the range of $[-1,1]$, is depicted in Fig.3(a). And from the Fig.3(b), we can see that the JS divergence is on the symmetry of $a=0.1$ and $x=0.5$.It once again proves that the JS divergence is symmetrical.In addition, Fig.3(c) show that when $a=0$, we have the minimum JS divergence and the two imaginary parts are same.

In total, the only condition that JS divergence is equal is that the real parts and the imaginary parts are equal at the same time.

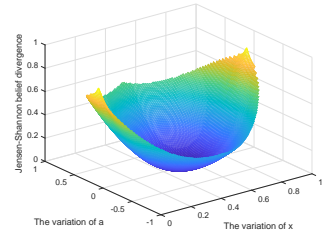

(a) The value of JS

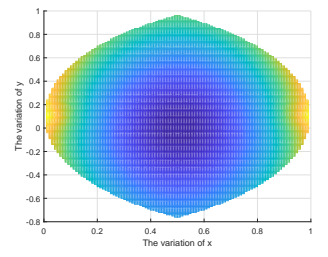

(b) Variations in $x$ and $y$

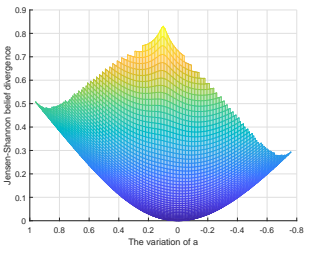

(c) JS as y varies

Figure 3: JS as CBBAs vary in the real and imaginary part

\section{Application}

Recently, the research of pattern recognition has attracted many researcher$\mathrm{s}^{\prime}$ attention. And in this part, a decision making algorithm based on CBD measure for pattern recognition is designed. The practicability of the algorithm is verified by its application in medical diagnosis.In addition, the ex- 
tension of the algorithm and the related work are analyzed and compared to verify the effectiveness of the algorithm.

\subsection{Decision-Making Algorithm}

We define that $X=\left\{x_{1}, \ldots, x_{i}, \ldots, x_{n}\right\}$ represents attributes,$P=\left\{P_{1}, \ldots, P_{j}, \ldots, P_{m}\right\}$ represents medical patterns in CBBAs of the form $P_{j}=\left\{<x_{i}, \mathbb{M}_{P_{j}}^{x_{i}}(Y), \mathbb{M}_{P_{j}}^{x_{i}}(N), \mathbb{M}_{P_{j}}^{x_{i}}(Y, N)>\right.$ $\left.\mid x_{i} \in X\right\}$, and $S=\left\{S_{1}, \ldots, S_{k}, \ldots S_{\lambda}\right\}$ represents the goal of pattern recognition in CBBAs of the form $S_{k}=\left\{<x_{i}, \mathbb{M}_{S_{k}}^{x_{i}}(Y), \mathbb{M}_{S_{k}}^{x_{i}}(N), \mathbb{M}_{S_{k}}^{x_{i}}(Y, N)>\mid x_{i} \in\right.$ $X\}$. The goal is to partition the sample $S=\left\{S_{1}, \ldots, S_{k}, \ldots S_{\lambda}\right\}$ according to a given $P=\left\{P_{1}, \ldots, P_{j}, \ldots, P_{m}\right\}$.

1. The distance between a given pattern $P_{j}$ and sample $S_{k}$ measured using the $\mathrm{CBD} J S_{C B B A}$

$$
\begin{aligned}
& J S\left(\mathbb{M}_{P_{j}}|| \mathbb{M}_{S_{k}}\right)=\frac{1}{n} \times \sum_{i=1}^{n} J S\left(\mathbb{M}_{P_{j}}^{x_{i}}|| \mathbb{M}_{S_{k}}^{x_{i}}\right) \\
& =\frac{1}{2} \sum_{i=1}^{n} K L\left(\mathbb{M}_{P_{j}}^{x_{i}} \| \frac{\mathbb{M}_{P_{j}}^{x_{i}}+\mathbb{M}_{S_{k}}^{x_{i}}}{2}\right)+\frac{1}{2} \sum_{i=1}^{n} K L\left(\mathbb{M}_{S_{k}}^{x_{i}} \| \frac{\mathbb{M}_{P_{j}}^{x_{i}}+\mathbb{M}_{S_{k}}^{x_{i}}}{2}\right)
\end{aligned}
$$

2. Choose the minimum distance between the CBBAs $\mathbb{M}_{S_{k}}$ and $\mathbb{M}_{P_{j}}$

$$
J S\left(\mathbb{M}_{P_{\mu}}|| \mathbb{M}_{S_{k}}\right)=\min _{1 \leq j \leq m} J S\left(\mathbb{M}_{P_{j}}|| \mathbb{M}_{S_{k}}\right)
$$

3. Sample $S_{k}$ is classified as $P_{\mu}$, where

$$
\begin{gathered}
\mu=\arg \min _{1 \leq j \leq m} J S\left(\mathbb{M}_{P_{j}} \| \mathbb{M}_{S_{k}}\right) \\
P_{\mu} \rightarrow S_{k}
\end{gathered}
$$


Table 1: Three patterns and a sample

\begin{tabular}{|c|c|c|c|c|}
\hline & Attributes & $\mathbf{M}_{Y}$ & $\mathbb{M}_{N}$ & $\mathbb{M}_{Y, N}$ \\
\hline \multirow{3}{*}{ Pattern1 } & $X 1$ & $0.9901 e^{\operatorname{iarctan}(0.0101)}$ & 0 & $0.0141 e^{\text {iarctan}}(-1.0000)$ \\
\hline & $X 2$ & $0.8062 e^{\operatorname{iarctan}(0.1250)}$ & 0 & $0.2236 e^{\operatorname{iarctan}(-0.5000)}$ \\
\hline & $X 3$ & $0.7071 e^{\operatorname{iarctan}(0.1429)}$ & $0.1118 e^{\operatorname{iarctan}(-0.5000)}$ & $0.2062 e^{\operatorname{iarctan}(-0.2500)}$ \\
\hline \multirow{3}{*}{ Pattern2 } & $X 1$ & $0.99055 e^{\operatorname{iarctan}(0.1111)}$ & $0.1414 e^{\operatorname{iarctan}(-1.0000)}$ & 0 \\
\hline & $X 2$ & $0.9901 e^{\operatorname{iarctan}(0.0101)}$ & 0 & $0.0141 e^{\operatorname{iarctan}(-1.0000)}$ \\
\hline & $X 3$ & $0.9055 e^{\operatorname{iarctan}(0.1111)}$ & 0 & $0.1414 e^{\operatorname{iarctan}(-1.0000)}$ \\
\hline \multirow{3}{*}{ Pattern3 } & $X 1$ & $0.6083 e^{\operatorname{iarctan}(0.1667)}$ & $0.2062 e^{\operatorname{iarctan}(-0.2500)}$ & $0.2062 e^{\operatorname{iarctan}(-0.2500)}$ \\
\hline & $X 2$ & $0.8062 e^{\operatorname{iarctan}(0.1250)}$ & 0 & $0.2236 e^{\operatorname{iarctan}(-0.5000)}$ \\
\hline & $X 3$ & $0.9901 e^{\operatorname{iarctan}(0.0101)}$ & 0 & $0.0141 e^{\operatorname{iarctan}(-1.0000)}$ \\
\hline \multirow{3}{*}{ Sample1 } & $X 1$ & $0.5099 e^{\operatorname{iarctan}(0.2000)}$ & $0.3041 e^{\operatorname{iarctan}(-0.1667)}$ & $0.2062 e^{\operatorname{iarctan}(-0.2500)}$ \\
\hline & $X 2$ & $0.6083 e^{\operatorname{iarctan}(0.1667)}$ & $0.2062 e^{\operatorname{iarctan}(-0.2500)}$ & $0.2062 e^{\operatorname{iarctan}(-0.2500)}$ \\
\hline & $X 3$ & $0.8062 e^{\operatorname{iarctan}(0.1250)}$ & $0.1118 e^{\operatorname{iarctan}(-0.5000)}$ & $0.1118 e^{\operatorname{iarctan}(-0.5000)}$ \\
\hline
\end{tabular}

\subsection{Application in a medical diagnosis}

Suppose a pattern recognition problem given pattern CBBAs $P=\left\{P_{1}, P_{2}, P_{3}\right\}$

and a kind of test sample CBBAs $S$, which relates to three kinds of attributes $\left\{x_{1}, x_{2}, x_{3}\right\}$ shown in Table 1 . The goal is to determine which of the given pattern $\left\{P_{1}, P_{2}, P_{3}\right\}$ is best suited to test sample $S$. The calculation process is described below.

1. The distance between a given pattern $P_{1}, P_{2}$, and $P_{3}$ and sample $S_{k}$ measured using the $\mathrm{CBD} J S_{C B B A}$, as follows:

$$
\begin{aligned}
& J S\left(\mathbb{M}_{P_{1}}|| \mathbb{M}_{S}\right)=0.119 ; \\
& J S\left(\mathbb{M}_{P_{2}}|| \mathbb{M}_{S}\right)=0.117 ; \\
& J S\left(\mathbb{M}_{P_{3}}|| \mathbb{M}_{S}\right)=0.066 ;
\end{aligned}
$$

2. The minimum value is between the sample $\mathbb{M}_{S_{k}}$ and $\mathbb{M}_{P_{3}}$

$$
J S\left(\mathbb{M}_{P_{3}}|| \mathbb{M}_{S}\right)=0.066 ;
$$

3. Sample $S$ is classified as $P_{3}$ 


$$
\begin{aligned}
& \mu=3 ; \\
& P_{3} \rightarrow S
\end{aligned}
$$

After above algorithm running, the results are presented in Table.2. As is obvious from step.2, $P_{3}$ is the smallest distance from $S$, so the sample $S$ is assigned to pattern $P_{3}$.

\subsection{Extension and Comparison}

In Section 6.2, the weight of each feature is evenly distributed, so an extended algorithm is proposed to include a weight ratio of each attributes.

$$
\begin{aligned}
& J S^{\omega}\left(\mathbb{M}_{P_{j}} \| \mathbb{M}_{S_{k}}\right)=\sum_{i=1}^{n} \omega_{i} J S\left(\mathbb{M}_{P_{j}}^{x_{i}} \| \mathbb{M}_{S_{k}}^{x_{i}}\right) \\
& =\sum_{i=1}^{n} \omega_{i} K L\left(\mathbb{M}_{P_{j}}^{x_{i}} \| \frac{\mathbb{M}_{P_{j}}^{x_{i}}+\mathbb{M}_{S_{k}}^{x_{i}}}{2}\right)+\sum_{i=1}^{n} \omega_{i} K L\left(\mathbb{M}_{S_{k}}^{x_{i}} \| \frac{\mathbb{M}_{P_{j}}^{x_{i}}+\mathbb{M}_{S_{k}}^{x_{i}}}{2}\right)
\end{aligned}
$$

where $\sum_{i=1}^{n} \omega_{i}=1$. $\omega_{i}$ can be considered as a subjective or objective factors.

On this basis, the validity of the two methods of average weight distance measurement is further verified, so we compare them with Garg and Rani method and[76] Xiao method[63].

In the medical diagnosis application, the frame of discernment has two elements, namely, $Y$ and $N . \mathbb{M}(Y), \mathbb{M}(N), \mathbb{M}(Y, N)$, three complex intuitionistic fuzzy sets, respectively relate to the membership, nonmembership, and hesitancy degrees. In [76], the weights $\omega_{i}$ are set to $\omega_{1}=0.3, \omega_{2}=$ $0.35, \omega_{3}=0.35$. After the calculation is completed, the results are presented in the Table., it is obvious that $J S^{\omega}\left(\mathbb{M}_{P_{3}}|| \mathbb{M}_{S}\right) \leq J S^{\omega}\left(\mathbb{M}_{P_{2}}|| \mathbb{M}_{S}\right) \leq$ $J S^{\omega}\left(\mathbb{M}_{P_{1}}|| \mathbb{M}_{S}\right)$. The conclusion is that pattern $P_{3}$ belongs to the sample $S$.

In [76], $<K_{1}, K_{2}, K_{3}, K_{4}>$ four processed data correlation coefficients with Garg and Rani method [76] and $d_{C B B A}, d_{C B B A}^{\omega}$ with Xiao method [63], the 
Table 2: Results generated by various method

\begin{tabular}{lllll}
\hline Methods & $\left(\mathbb{M}_{P_{1}}|| \mathbb{M}_{S}\right)$ & $\left(\mathbb{M}_{P_{2}}|| \mathbb{M}_{S}\right)$ & $\left(\mathbb{M}_{P_{3}}|| \mathbb{M}_{S}\right)$ & $\begin{array}{l}\text { Classification } \\
\text { results }\end{array}$ \\
\hline$\left(d_{C B B A}\right)$ & 0.2256 & 0.2436 & 0.1526 & $P_{3}$ \\
$d_{C B B A}^{\omega}$ & 0.2156 & 0.2400 & 0.1553 & $P_{3}$ \\
$K 1$ & 0.0907 & 0.0539 & 0.0249 & $P_{3}$ \\
$K 2$ & 0.2458 & 0.2941 & 0.1838 & $P_{3}$ \\
$K 3$ & 0.0868 & 0.0531 & 0.0255 & $P_{3}$ \\
$K 4$ & 0.2317 & 0.0531 & 0.0255 & $P_{3}$ \\
$J S$ & 0.1195 & 0.1175 & 0.0667 & $P_{3}$ \\
$J S^{\omega}$ & 0.1136 & 0.1171 & 0.0695 & $P_{3}$ \\
\hline
\end{tabular}

distance between complex intuitionistic fuzzy sets,are presented in Table 2. It is obvious that all of them have a result that $P_{3}$ belongs to the sample $S$ and the proposed method for pattern recognition based on $J S, J S^{\omega}$ as effective as them. Compared with Garg and Rani method [76], the proposed method can measure the distance when the frame of discernment has two or more elements. Compared with Xiao method [63], the proposed method can take the number of elements in the subset into account. This application shows that the CBD can be used in the real applications under uncertain environments.

\section{Conclusions}

In this paper, complex belief divergence (CBD), a novel divergence measure, is proposed for complex mass function. This is the first time that divergence measure is studied in the realm of complex mass function. The properties of the CBD is analysed. The effectiveness of the CBD is illustrated by numerical examples. Finally, an application is proposed based on $\mathrm{CBD}$. In the future, we will investigate on how to use CBD in many 
more complex applications. Also, the relationship of CBD and other divergence measures, like Rényi divergence and Tsallis divergence, will be also explored.

\section{Declarations}

\subsection{Conflict of interest}

The authors declare that they have no conflict of interest.

\subsection{Ethical approval}

This article does not contain any studies with human participants or animals performed by any of the authors.

\section{References}

[1] Y. Deng, Uncertainty measure in evidence theory, SCIENCE CHINA Information Sciences 64 (2021) 10.1007/s11432-020-3006-9.

[2] F. Xiao, Z. Zhang, J. Abawajy, Workflow scheduling in distributed systems under fuzzy environment, Journal of Intelligent \& Fuzzy Systems 37 (4) (2019) 5323-5333.

[3] D. Meng, M. Liu, S. Yang, H. Zhang, R. Ding, A fluid-structure analysis approach and its application in the uncertainty-based multidisciplinary design and optimization for blades, Advances in Mechanical Engineering 10 (6) (2018) 1687814018783410.

[4] X. Deng, W. Jiang, On the negation of a Dempster-Shafer belief structure based on maximum uncertainty allocation, Information Sciences 516 (2020) 346-352. 
[5] F. Xiao, EFMCDM: Evidential fuzzy multicriteria decision making based on belief entropy, IEEE Transactions on Fuzzy Systems 28 (7) (2020) 1477-1491.

[6] Y. Song, X. Wang, W. Wu, W. Quan, W. Huang, Evidence combination based on credibility and non-specificity, Pattern Analysis and Applications 21 (1) (2018) 167-180.

[7] W. Jiang, Y. Cao, X. Deng, A novel Z-network model based on Bayesian network and Z-number, IEEE Transactions on Fuzzy Systems (2019) DOI: 10.1109/TFUZZ.2019.2918999.

[8] Y. Tian, L. Liu, X. Mi, B. Kang, ZSLF: A new soft likelihood function based on Z-numbers and its application in expert decision system, IEEE Transactions on Fuzzy Systems (2020) DOI: 10.1109/TFUZZ.2020.2997328.

[9] L. Fei, Y. Feng, L. Liu, On Pythagorean fuzzy decision making using soft likelihood functions, International Journal of Intelligent Systems 34 (12) (2019) 3317-3335.

[10] J. Yang, S. Li, Z. Xu, H. Liu, W. Yao, An understandable way to extend the ordinary linear order on real numbers to a linear order on interval numbers, IEEE Transactions on Fuzzy Systems (2020) DOI: 10.1109/TFUZZ.2020.3006557.

[11] J. C. Alcantud, F. Feng, R. Yager, An N-soft set approach to rough sets, IEEE Transactions on Fuzzy Systems (2019) DOI: 10.1109/TFUZZ.2019.2946526. 
[12] W. Jiang, K. Huang, J. Geng, X. Deng, Multi-scale metric learning for few-shot learning, IEEE Transactions on Circuits and Systems for Video Technology (2020) DOI: 10.1109/TCSVT.2020.2995754.

[13] P. Liu, X. Zhang, A new hesitant fuzzy linguistic approach for multiple attribute decision making based on Dempster-Shafer evidence theory, Applied Soft Computing 86 (2020) 105897.

[14] S. Mao, Y. Han, Y. Deng, D. Pelusi, A hybrid DEMATEL-FRACTAL method of handling dependent evidences, Engineering Applications of Artificial Intelligence 91 (2020) Article Number UNSP 103543. doi:\{10.1016/j.engappai.2020.103543\}.

[15] F. Xiao, GIQ: A generalized intelligent quality-based approach for fusing multi-source information, IEEE Transactions on Fuzzy Systems (2020) DOI: 10.1109/TFUZZ.2020.2991296.

[16] L. Fei, Y. Deng, Multi-criteria decision making in Pythagorean fuzzy environment, Applied Intelligence 50 (2) (2020) 537-561.

[17] M. Tang, H. Liao, E. Herrera-Viedma, C. P. Chen, W. Pedrycz, A dynamic adaptive subgroup-to-subgroup compatibility-based conflic$\mathrm{t}$ detection and resolution model for multicriteria large-scale group decision making, IEEE Transactions on Cybernetics (2020) DOI: 10.1109/TCYB.2020.2974924.

[18] L. Fei, Y. Feng, L. Liu, Evidence combination using OWA-based soft likelihood functions, International Journal of Intelligent Systems 34 (9) (2019) 2269-2290. 
[19] Z. Liu, Q. Pan, J. Dezert, J.-W. Han, Y. He, Classifier fusion with contextual reliability evaluation, IEEE Transactions on Cybernetics 48 (5) (2018) 1605-1618.

[20] Y.-F. Li, H.-Z. Huang, J. Mi, W. Peng, X. Han, Reliability analysis of multi-state systems with common cause failures based on bayesian network and fuzzy probability, Annals of Operations Research (2019) $1-15$.

[21] H. Zhang, D. Meng, Y. Zong, F. Wang, T. Xin, A modeling and analysis strategy of constellation availability using on-orbit and ground added launch backup and its application in the reliability design for a remote sensing satellite, Advances in Mechanical Engineering 10 (4) (2018) 1687814018769783.

[22] J. Mi, M. Beer, Y.-F. Li, M. Broggi, Y. Cheng, Reliability and importance analysis of uncertain system with common cause failures based on survival signature, Reliability Engineering \& System Safety (2020) 106988.

[23] F. Xiao, A distance measure for intuitionistic fuzzy sets and its application to pattern classification problems, IEEE Transactions on Systems, Man, and Cybernetics: Systems (2019) DOI: 10.1109/TSMC.2019.2958635.

[24] Z.-G. Liu, Q. Pan, J. Dezert, A. Martin, Combination of classifiers with optimal weight based on evidential reasoning, IEEE Transactions on Fuzzy Systems 26 (3) (2018) 1217-1230.

[25] S. M. Hatefi, S. M. Moshashaee, I. Mahdavi, A bi-objective program- 
ming model for reliable supply chain network design under facility disruption, International Journal of Integrated Engineering 11 (6) (2019) 80-92.

[26] T. Wen, Y. Deng, Identification of influencers in complex networks by local information dimensionality, Information Sciences 512 (2020) 549562.

[27] D. Pelusi, R. Mascella, L. Tallini, J. Nayak, B. Naik, A. Abraham, Neural network and fuzzy system for the tuning of gravitational search algorithm parameters, Expert Systems with Applications 102. doi:10.1016/j.eswa.2018.02.026.

[28] Z. Cao, C.-H. Chuang, J.-K. King, C.-T. Lin, Multi-channel EEG recordings during a sustained-attention driving task, Scientific Data 6 (2019) DOI: $10.1038 /$ s41597-019-0027-4.

[29] Z. Cao, W. Ding, Y.-K. Wang, F. K. Hussain, A. Al-Jumaily, C.-T. Lin, Effects of repetitive SSVEPs on EEG complexity using multiscale inherent fuzzy entropy, Neurocomputing 389 (2019) 198-206.

[30] C. Fu, M. Xue, W. Chang, D. Xu, S. Yang, An evidential reasoning approach based on risk attitude and criterion reliability, KnowledgeBased Systems 199 (2020) 105947.

[31] Y. Pan, L. Zhang, X. Wu, M. J. Skibniewski, Multi-classifier information fusion in risk analysis, Information Fusion 60 (2020) 121-136.

[32] Z. Luo, Y. Deng, A vector and geometry interpretation of basic probability assignment in Dempster-Shafer theory, International Journal of Intelligent Systems 35 (6) (2020) 944-962. 
[33] X. Deng, W. Jiang, Z. Wang, Zero-sum polymatrix games with link uncertainty: A Dempster-Shafer theory solution, Applied Mathematics and Computation 340 (2019) 101-112.

[34] X. Wang, Y. Song, Uncertainty measure in evidence theory with its applications, Applied Intelligence 48 (7) (2018) 1672-1688.

[35] D. Cavaliere, J. A. Morente-Molinera, V. Loia, S. Senatore, E. HerreraViedma, Collective scenario understanding in a multi-vehicle system by consensus decision making, IEEE Transactions on Fuzzy Systems (2019) DOI: 10.1109/TFUZZ.2019.2928787.

[36] R. Fang, H. Liao, J.-B. Yang, D.-L. Xu, Generalised probabilistic linguistic evidential reasoning approach for multi-criteria decisionmaking under uncertainty, Journal of the Operational Research Society (2) (2020) 1-15.

[37] J. Ma, W. Liu, P. Miller, H. Zhou, An evidential fusion approach for gender profiling, Information Sciences 333 (2016) 10-20.

[38] X. Xu, J. Zheng, J.-b. Yang, D.-1. Xu, Y.-w. Chen, Data classification using evidence reasoning rule, Knowledge-Based Systems 116 (2017) 144-151.

[39] F. Xiao, Z. Cao, A. Jolfaei, A novel conflict measurement in decision making and its application in fault diagnosis, IEEE Transactions on Fuzzy Systems (2020) DOI: 10.1109/TFUZZ.2020.3002431.

[40] F. Liu, X. Gao, J. Zhao, Y. Deng, Generalized belief entropy and its application in identifying conflict evidence, IEEE Access 7 (2019) 126625126633. 
[41] F. Xiao, Evidence combination based on prospect theory for multi-sensor data fusion, ISA Transactions (2020) DOI: $10.1016 /$ j.isatra.2020.06.024.

[42] M. Zhou, X.-B. Liu, Y.-W. Chen, X.-F. Qian, J.-B. Yang, J. Wu, Assignment of attribute weights with belief distributions for MADM under uncertainties, Knowledge-Based Systems 189 (2020) 105110.

[43] Z. Luo, Y. Deng, A matrix method of basic belief assignment's negation in Dempster-Shafer theory, IEEE Transactions on Fuzzy Systems 27 (2019) 10.1109/TFUZZ.2019.2930027.

[44] Z. Liu, X. Zhang, J. Niu, J. Dezert, Combination of classifiers with different frames of discernment based on belief functions, IEEE Transactions on Fuzzy Systems (2020) DOI: 10.1109/TFUZZ.2020.2985332.

[45] H. Yan, Y. Deng, An improved belief entropy in evidence theory, IEEE Access 8 (1) (2020) 57505-57516.

[46] X. Xu, H. Xu, C. Wen, J. Li, P. Hou, J. Zhang, A belief rule-based evidence updating method for industrial alarm system design, Control Engineering Practice 81 (2018) 73-84.

[47] C. Fu, W. Chang, S. Yang, Multiple criteria group decision making based on group satisfaction, Information Sciences 518 (2020) 309-329.

[48] B. Kang, P. Zhang, Z. Gao, G. Chhipi-Shrestha, K. Hewage, R. Sadiq, Environmental assessment under uncertainty using Dempster-Shafer theory and Z-numbers, Journal of Ambient Intelligence and Humanized Computing (2019) DOI: 10.1007/s12652-019-01228-y. 
[49] X.-B. Xu, X. Ma, C.-L. Wen, D.-R. Huang, J.-N. Li, Self-tuning method of PID parameters based on belief rule base inference, Information Technology And Control 47 (3) (2018) 551-563.

[50] W. Jiang, Z. Zhang, X. Deng, A novel failure mode and effects analysis method based on fuzzy evidential reasoning rules, IEEE Access 7 (2019) 113605-113615.

[51] C. Fu, B. Hou, W. Chang, N. Feng, S. Yang, Comparison of evidential reasoning algorithm with linear combination in decision making, International Journal of Fuzzy Systems 22 (2) (2020) 686-711.

[52] M. Zhou, X. Liu, J. Yang, Evidential reasoning approach for MAD$\mathrm{M}$ based on incomplete interval value, Journal of Intelligent \& Fuzzy Systems 33 (6) (2017) 3707-3721.

[53] M. Zhou, X.-B. Liu, Y.-W. Chen, J.-B. Yang, Evidential reasoning rule for MADM with both weights and reliabilities in group decision making, Knowledge-Based Systems 143 (2018) 142-161.

[54] X. Deng, W. Jiang, A total uncertainty measure for D numbers based on belief intervals, International Journal of Intelligent Systems 34 (12) (2019) 3302-3316.

[55] P. Liu, X. Zhang, Z. Wang, An extended VIKOR method for multiple attribute decision making with linguistic D numbers based on fuzzy entropy, International Journal of Information Technology and Decision Making 19 (1) (2020) 143-167.

[56] L. Pan, Y. Deng, Probability transform based on the ordered weighted 
averaging and entropy difference, International Journal of Computers Communications \& Control 15 (4) (2020) 3743.

[57] Q. Cai, X. Gao, Y. Deng, Pignistic belief transform: A new method of conflict measurement, IEEE Access 8 (1) (2020) 15265-15272.

[58] F. Xiao, Generalization of Dempster-Shafer theory: A complex mass function, Applied Intelligence (2019) DOI: 10.1007/s10489-019_ 01617-y.

[59] F. Xiao, Generalized belief function in complex evidence theory, Journal of Intelligent \& Fuzzy Systems 38 (4) (2020) 3665-3673.

[60] X. Gao, Y. Deng, Quantum Model of Mass Function, International Journal of Intelligent Systems 35 (2) (2020) 267-282.

[61] A. P. Dempster, Upper and lower probabilities induced by a multivalued mapping, Annals of Mathematical Statistics 38 (2) (1967) 325-339.

[62] G. Shafer, A mathematical theory of evidence, Technometrics 20 (1) (1978) 242.

[63] F. Xiao, CED: A distance for complex mass functions, IEEE Transactions on Neural Networks and Learning Systems (2020) DOI: 10.1109/TNNLS.2020.2984918.

[64] V. Torra, Y. Narukawa, M. Sugeno, On the f-divergence for discrete non-additive measures, Information Sciences 512 (2020) 50-63.

[65] Y. Song, Q. Fu, Y.-F. Wang, X. Wang, Divergence-based cross entropy and uncertainty measures of Atanassov's intuitionistic fuzzy sets with 
their application in decision making, Applied Soft Computing 84 (2019) 105703.

[66] G. Squillero, A. Tonda, Divergence of character and premature convergence: A survey of methodologies for promoting diversity in evolutionary optimization, Information Sciences 329 (2016) 782-799.

[67] Nancy, H. Garg, A novel divergence measure and its based TOPSIS method for multi criteria decision-making under single-valued neutrosophic environment, Journal of Intelligent and Fuzzy Systems 36 (1) (2019) 101-115.

[68] Q. Zhou, H. Mo, Y. Deng, A new divergence measure of Pythagorean Fuzzy sets based on belief function and its application in medical diagnosis, Mathematics 8 (1) (2020) 10.3390/math8010142.

[69] Q. Xu, Y. Liu, X. Li, Z. Yang, J. Wang, M. Sbert, R. Scopigno, Browsing and exploration of video sequences: A new scheme for key frame extraction and 3D visualization using entropy based Jensen divergence, Information Sciences 278 (2014) 736-756.

[70] I. Loh, G. Phelan, Dimensionality and disagreement: Asymptotic belief divergence in response to common information, International Economic Review 60 (4) (2019) 1861-1876.

[71] L. Fei, Y. Deng, A new divergence measure for basic probability assignment and its applications in extremely uncertain environments, International Journal of Intelligent Systems 34 (4) (2019) 584-600.

[72] S. Kullback, R. A. Leibler, On Information and Sufficiency, Annals of Mathematical Statistics 22 (1) 79-86. 
[73] B. Kang, Y. Deng, The maximum Deng entropy, IEEE Access 7 (1) (2019) 120758-120765.

[74] X. Gao, Y. Deng, The pseudo-pascal triangle of maximum Deng entropy, International Journal of Computers Communications \& Control 15 (1) (2020) 1006.

[75] Y. Song, Y. Deng, Divergence measure of belief function and its application in data fusion, IEEE Access 7 (2019) 107465-107472.

[76] H. Garg, D. Rani, A robust correlation coefficient measure of complex intuitionistic fuzzy sets and their applications in decision-making, Applied Intelligence 49 (2) (2019) 496-512. 